\title{
Mutations in the Ankyrin Repeat Domain of the Human TRPV4 Protein Affect Binding of ATP: a Combined Molecular Dynamics and Network Analysis Study
}

\author{
Ying Sun ${ }^{1}$, Wei Liu ${ }^{1}$, Ji Lv², Shao-Hui Liu ${ }^{1}$, and Ya Kun Chen ${ }^{1}$ \\ ${ }^{1}$ Jilin University \\ ${ }^{2}$ Chinese Academy of Sciences
}

November 6, 2020

\begin{abstract}
Molecular dynamics (MD) simulations and residue interaction network analyses were carried out to investigate how some mutations on the human TRPV4 ankyrin repeating domain (ARD) change the ATP-binding strengths. Contact residue interaction networks (RINs) generated from the MD simulation trajectories are connected graphs with the ATP nodes having high importance. Besides four ATP-binding residues identified in early researches, extra ATP-binding residues are found for the wild type and mutant human TRPV4 ARDs based on the contact RIN results. Analyses of the first neighbor lists of the ATP nodes in contact RINs, and visualization of the average structures help to locate two distinct ATP-binding areas for different groups of mutants, with the wild type, K197R, R269H, V342F mutants in the first group, and E183K, E278K and R232C in the second. Further dihedral angle analyses, PCA analyses and mutation-site first-neighbor analyses demonstrate that the mutations usually cause remote interaction changes to the mutation sites, alter the collaborative behaviors of different domains of the human TRPV4 ARD, and eventually affect the ATP-binding strengths.
\end{abstract}

\section{Hosted file}

with_authors.pdf available at https://authorea.com/users/373689/articles/491302-mutationsin-the-ankyrin-repeat-domain-of-the-human-trpv4-protein-a\%EF\%AC\%80ect-binding-of-atp-acombined-molecular-dynamics-and-network-analysis-study 
figures/Figure1/Figure1-eps-converted-to.pdf 
figures/Figure2/Figure2-eps-converted-to.pdf 
figures/Figure3/Figure3-eps-converted-to.pdf 
figures/Figure4/Figure4-eps-converted-to.pdf 
figures/Figure5/Figure5-eps-converted-to.pdf 